\title{
Corneal Elevation Values in Normal Eyes, forme fruste Keratoconus and Keratoconus at Different Stages Measured by Scheimpflug Imaging
}

\author{
${ }^{1}$ Maria A Henriquez, ${ }^{2}$ Luis Izquierdo Jr, ${ }^{3}$ David Dañin
}

\begin{abstract}
Purpose: To compare corneal elevation values in normal eyes, forme fruste keratoconus (FFKC) and different stages of keratoconus using Scheimpflug imaging.

Materials and methods: This prospective, comparative study included 267 eyes (107 normal eyes, 21 FFKC and 139 keratoconus). Keratoconic eyes were divided into four groups according to keratometry values. Maximum posterior elevation (PE) above the (best fit sphere (BFS) at the central $5 \mathrm{~mm}$ were measured using the Pentacam (Oculus Optikgeräte $\mathrm{GmbH}$ ). Receiver operating characteristic curves were used to determine the test's overall predictive accuracy and to identify optimal cutoff points to discriminate between the groups.
\end{abstract}

Results: PE had the smallest values in normal eyes and increased in FFKC and each progressive stage of keratoconus. Mean $\mathrm{PE}$ was $9.98 \pm 5.33 \mu \mathrm{m}$ in normal eyes, $18.09 \pm 9.23 \mu \mathrm{m}$ in FFKC and $24.97 \mu \mathrm{m} \pm 15.89,37.82 \pm 18.64,46.82 \pm 21.41$ and $66.07 \pm 39.09$, in keratoconus stage I, II, III and IV respectively.

Conclusion: Posterior elevation values increased according to the severity of keratoconus disease. PE can be used as indicator of keratoconus progression.

Keywords: Keratoconus, Scheimpflug imaging, Posterior elevation, Amsler-Krumeich.

How to cite this article: Henriquez MA, Izquierdo L Jr, Dañin D. Corneal Elevation Values in Normal Eyes, forme fruste Keratoconus and Keratoconus at Different Stages Measured by Scheimpflug Imaging. Int J Kerat Ect Cor Dis 2014;3(1):36-39.

Source of support: Nil

Conflict of interest: None declared

\section{INTRODUCTION}

Keratoconus is a slowly progressive disorder in which the cornea assumes an irregular conical shape as a result of central or paracentral noninflammatory thinning of the cornea.

\footnotetext{
${ }^{1,2}$ Professor, ${ }^{3}$ Fellow

${ }^{1-3}$ Department of Research, Oftalmosalud Instituto de Ojos Lima, Peru

Corresponding Author: Maria A Henriquez, Professor Department of Research, Oftalmosalud Instituto de Ojos, AV Javier Prado Este 1142, San Isidro, Lima 27, Peru, Phone: 005115121300 or 005112254444 , e-mail: mariale_1610@ hotmail.com
}

The corneal thinning induces irregular astigmatism, myopia and protrusion, causing mild to severe impairment of vision. Keratoconus tends to be bilateral, asymmetric, corneal ectatic and enantiomorphic disease ${ }^{1}$ and the different stages of keratoconus present different refractive, topographic, and slitlamp microscopy signs. ${ }^{2,3}$ Early diagnosis of this disease, especially in the screening of refractive surgery candidates, could prevent postoperative complications in these patients. ${ }^{4-8}$

The Pentacam Comprehensive Eye Scanner is based on a capture method called Scheimpflug imaging, which measures 25,000 elevation points and gives a representation of the shape of the corneal surface. The Pentacam compares the raw elevation data against a common shape-namely, a best fit sphere (BFS)-giving a posterior surface topography. Measurements of corneal thickness and posterior elevation (PE) with the Pentacam are well-investigated and highly reproducible and repeatable., ${ }^{9,10}$

The characteristic stromal thinning in keratoconus corresponds to an increased PE above the BFS on corneal topography. Studies have investigated posterior corneal elevation values in keratoconic, subclinically keratoconic, forme fruste keratoconus (FFKC) and normal corneas using the Pentacam. ${ }^{11-13}$ However, little has been published comparing this value in keratoconic corneas at different stages. ${ }^{14,15}$

The aim of this study was to measure posterior corneal elevation values in normal corneas, FFKC and keratoconus at different stages of the disease.

\section{PATIENTS AND METHODS}

In this prospective, observational, comparative study, a single cornea specialist examined 267 eyes of 267 consecutive patients at the Cornea Service of the Instituto de Ojos Oftalmo Salud, Lima, Peru, between April 2007 and September 2009.

On the basis of clinical signs, tangential placido diskbased videokeratography evaluation and Scheimpflug imaging, we employed the following classification scheme.

Normal eyes: These eyes were from patients who had bilateral normal corneas, with no ocular pathology, no irregular corneal pattern, no previous ocular surgery, no signi- 
ficant refractive error, and no clinical signs of keratoconus or scissoring on retinoscopy. The Pentacam reported them as normal. This group included unilateral eyes that were randomly selected.

Forme fruste keratoconus (FFKC): This group included topographically normal eyes of patients with keratoconus in the other eye (by topography and Pentacam).

Keratoconus $(\mathrm{KC})$ : This group was defined on the basis of classic corneal biomicroscopic and topographic characteristics, ${ }^{1,16-18}$ as the presence of $\geq 1$ clinical signs (corneal stromal thinning, Vogt striae, Fleischer ring, scissoring of the red reflex, or oil droplet sign identified by retinoscopy) and topographic evaluation (an increased area of corneal power surrounded by concentric areas of decreasing power, inferior-superior power asymmetry, and skewing of the steepest radial axes above and below the horizontal meridian). Keratoconus patients with a history of corneal operation or with extensive corneal scarring were excluded, as were contact lens-wearing patients who showed corneal warping. ${ }^{18}$ Contact lens use was discontinued at least 3 weeks (rigid lenses) or 1 week (soft lenses) before assessment. Keratoconus group was subdivided into four groups according to the keratometry of Amsler-Krumeich classification: ${ }^{3}$

Keratoconus stage I (KC-I): Mean central $\mathrm{K}$ readings $\leq 48$ D.

Keratoconus stage II (KC-II): Mean central $\mathrm{K}$ readings $>48$ D to $\leq 53$ D.

Keratoconus stage III (KC-III): Mean central K readings $>53 \mathrm{D}$ to $\leq 55 \mathrm{D}$.

Keratoconus stage $I V(K C-I V)$ : Mean central $\mathrm{K}$ readings $>55$ D.

A full ophthalmological examination, including uncorrected visual acuity, spherical equivalent refraction, best spectacle-corrected visual acuity, slit-lamp microscopy, fundus examination, and rotating Scheimpflug imaging (Pentacam), was performed in all groups. Informed consent was obtained in accordance with the recommendations of the Declaration of Helsinki.

\section{Pentacam Analysis and Data Calculation}

For the Pentacam measurements, the patient sat in a chair, with the chin placed on the chin rest and the forehead put against the forehead strap in dim-light conditions. The patient was asked to look with both eyes open into the black spot in the middle of the instrument's blue fixation beam. When the image was focused, the instrument took the Scheimpflug images automatically.

Data were collected using Pentacam Comprehensive Eye Scanner software, version 1.17 r37. For posterior corneal elevation measurements, a best fit sphere (BFS) was used as a reference surface. The sphere that best fit the posterior corneal surface was automatically generated by the software, with the float option over a $8 \mathrm{~mm}$ fit. On these maps, PE were measured as the maximum values above the BFS in the central $5 \mathrm{~mm}$ of the posterior cornea.

\section{STATISTICAL ANALYSES}

We compared normal corneas vs keratoconic corneas (all stages), as well as normal corneas vs FFKC, in separate series of analyses. Receiver-operating characteristic (ROC) ${ }^{19}$ curves were obtained using SPSS 15 (SPSS Inc., Chicago, IL). These curves are obtained by plotting sensitivity against 1-specificity, calculated for each value observed. An area of $100 \%$ implies that the test perfectly discriminated between groups. Area under the ROC curve was calculated to describe the predictive accuracy of the different parameters and to determine the cutoff point for the most sensitive parameter. An t-test was used to determine whether the difference between eyes in keratoconic patients and normal subjects was significantly different. A p-value less than 0.05 was considered statistically significant.

\section{RESULTS}

A total of 267 eyes (267 patients) were included in this study. The control group consisted of 107 eyes of 107 individuals with normal corneas [50 males, 57 females; mean age \pm standard deviation (SD), $30.56 \pm 5.69$ years]. The FFKC group were 21 eyes of 21 patients (10 males, 11 females, mean age $31.44 \pm 6.84$ years). The keratoconus group consisted of 139 eyes of 139 patients: 50, 53, 22 and 14 eyes with keratoconus stages I, II, III and IV, respectively, mean age was $32.07 \pm 16.38$ years ( 72 women, 67 men). There were no statistically significant differences between the three groups in terms of age or sex $(p>0.05)$.

Table 1 shows the mean posterior elevation in all groups.

Table 2 shows the sensitivity and specificity of cutoff points, the area under the ROC curve, the standard error, and the $\mathrm{p}$-value for $\mathrm{PE}$ at different stage of keratoconus disease. The closer the value is to 1 (for the AUROC), means the highest sensitivity for discriminating FFKC and keratocons from normal eyes.

\section{DISCUSSION}

In the past few years, posterior elevation has been reported to be a good parameter to discriminate between normal and keratoconus eyes; however, mean posterior elevation values varies from author to author, as well cutoff points, are different in each report. There are some points that should be taken in consideration when posterior elevation is evaluated: the reference surface used, that could be, for 
Table 1: Mean posterior and standard deviation in normal, forme fruste keratoconus (FFKC), and keratoconus in all stages (KC-I-keratoconus stage I, KC-II—keratoconus stage II, KC-III-keratoconus stage III and KC-IV—keratoconus stage IV)

\begin{tabular}{lllllll}
\hline & Normal & FFKC & KC-I & KC-II & KC-III & KC-IV \\
\hline Mean PE & 9.98 & 18.09 & 24.97 & 37.82 & 46.82 & 66.07 \\
Standard deviation (SD) & 5.33 & 9.23 & 15.89 & 18.64 & 21.41 & 39.09 \\
\hline
\end{tabular}

Table 2: Sensitivity and specificity of cutoff points, the area under the receiver-operating characteristic curve, the standard error, and the $\mathrm{p}$-value for PE in normal eyes, forme fruste keratoconus, and keratoconus at different stage (KC-I-keratoconus stage I, KC-IIkeratoconus stage II, KC-III-keratoconus stage III, KC-IV—keratoconus stage IV)

\begin{tabular}{llllll}
\hline & Normal vs FFKC & Normal vs KC-I & Normal vs KC-II & Normal vs KC-III & Normal vs KC-IV \\
\hline Cutoff point $(\mu \mathrm{m})$ & 13.5 & 14.5 & 16.5 & 25.5 & 35.5 \\
Sensitivity & 0.71 & 0.81 & 0.85 & 0.98 & 0.97 \\
Specificity & 0.77 & 0.9 & 0.85 & 0.91 & 0.96 \\
AUROC & 0.78 & 0.91 & 0.93 & 0.99 & 0.99 \\
Standard error & 0.05 & 0.02 & 0.01 & 0.002 & 0.002 \\
p-value & $<0.001$ & $<0.001$ & $<0.001$ & $<0.001$ & $<0.001$ \\
\hline
\end{tabular}

example, an sphere (best fit sphere, BFS) or a toric aspheric surface (best fit toric and aspheric surface) $;^{20}$ the place were the posterior elevation is measured, that could be at the center of the cornea, at the thinnest point of the cornea or as the maximum values above the BFS in the central 5 or $9 \mathrm{~mm}$ of the posterior cornea and finally the keratoconus population evaluated, some studies included mild and moderate keratoconus, another's all types of keratoconus.

Using the Pentacam, Mihaltz et $\mathrm{a}^{12}$ described a cutoff value of $15.5 \mu \mathrm{m}$, with $95.1 \%$ sensitivity and $94.3 \%$ specificity; de Sanctis et al ${ }^{11}$ identified a cutoff value of $35 \mu \mathrm{m}$ for keratoconus, with $97.3 \%$ sensitivity and $96.9 \%$ specificity; and Ucakhan et $\mathrm{al}^{21}$ reported a cutoff point of $26.5 \mu \mathrm{m}$, with $97.7 \%$ sensitivity and $81 \%$ specificity for keratoconus. In our study, mean PE was $9.98 \pm 5.33 \mu \mathrm{m}$ in normal eyes, $18.09 \pm$ $9.23 \mu \mathrm{m}$ in FFKC, $24.97 \pm 15.89 \mu \mathrm{m}, 37.82 \pm 18.64 \mu \mathrm{m}, 46.82$ $\pm 21.41 \mu \mathrm{m}$ and $66.07 \pm 39.09 \mu \mathrm{m}$ in Keratoconus stage I, II, III and IV, respectively. Also, cutoff values were different in each keratoconus stage, having higher AUROC values when discriminating late disease from normal eyes, than when discriminating early disease of normal eyes. A cutoff point of $13.5 \mu \mathrm{m}$ had $71.9 \%$ sensitivity and $77.3 \%$ specificity (AUROC 0.78) discriminating normal eyes from FFKC, while a cutoff value of $35.5 \mu \mathrm{m}$ had an AUROC value of 0.99 , $97 \%$ sensitivity and $96 \%$ specificity discriminating normal eyes from keratoconus stage IV, showing that PE values increased with disease progression. In a recently article, ${ }^{22}$ we reported an intereye asymmetries on posterior elevation values, of $20.08 \mu \mathrm{m}$ in keratoconus patients, knowing that the majority of keratoconus patients have bilateral disease, and its presentation is asymmetric between the two eyes, this support our results were different stages of keratoconus showed different posterior elevation values.
This study suggests that $P E$ is effective in discriminating normal eyes from keratoconus, but it is effectively lower in FFKC and early disease. With Scheimpflug technology becoming more common place, and corneal collagen crosslinking used as treatment to half the progression of the keratoconus, we believe that PE should be incorporated into standard evaluation of keratoconus progression.

We conclude, from this study, that PE increases with each progressive stage of keratoconus and it is effectively discriminate between groups is lower in early keratoconus disease, compared with late keratoconus disease.

\section{ACKNOWLEDGMENT}

The authors thank Lic. Rocio P Falcon for statistical expertise.

\section{REFERENCES}

1. Rabinowitz YS. Keratoconus. Surv Ophthalmol 1998;42(4):297319.

2. Lovisolo CF, Calossi A, Ottone AC. Intrasomal Corneal Ring Segments. Canelli (Italy): Fabiano Editore; 2000. Intrastromal inserts in keratoconus and ectatic corneal conditions 2000;95163.

3. Alió JL, Shabayek MH. Corneal higher order aberrations: a method to grade keratoconus. J Refract Surg 2006;22(6):539545 .

4. Wilson SE, Klyce SD. Screening for corneal topographic abnormalities before refractive surgery. Ophthalmology 1994;101(1): 145-152.

5. Nesburn AB, Bahri S, Salz J, Rabinowitz YS, Maguen E, Hofbauer J, Berlin M, Macy JI. Keratoconus detected by videokeratography in candidates for photorefractive keratectomy. J Refract Surg 1995;11(3):194-201.

6. Seiler T, Quurke AW. Iatrogenic keratectasia after LASIK in a case of forme fruste keratoconus. J Cataract Refract Surg 1998; 24(9):1007-1009. 
7. Randleman JB, Russell B, Ward M, Thompson KP, Stulting $\mathrm{RD}$. Risk factors and prognosis for corneal ectasia after LASIK. Ophthalmology 2003;110(2):267-275.

8. Binder PS, Lindstrom RL, Stulting RD, Donnenfeld E, Wu H, McDonnell P, Rabinowitz Y. Keratoconus and corneal ectasia after LASIK. J Cataract Refract Surg 2005;31(1):2035-2038.

9. de Sanctis U, Missolungi A, Mutani B, Richiardi L, Grignolo FM. Reproducibility and repeatability of central corneal thickness measurement in keratoconus using the rotating Scheimpflug camera and ultrasound pachymetry. Am J Ophthalmol 2007; 144(5):712-718.

10. Chen D, Lam AK. Intrasession and intersession repeatability of the Pentacam system on posterior corneal assessment in the normal human eye. J Cataract Refract Surg 2007;33(3):448-454.

11. de Sanctis U, Loiacono C, Richiardi L, Turco D, Mutani B, Grignolo FM. Sensitivity and specificity of posterior corneal elevation measured by Pentacam in discriminating keratoconus/ subclinical keratoconus. Ophthalmology 2008;115(9):1534-1539.

12. Mihaltz K, Kovacs I, Takacs A, Nagy ZZ. Evaluation of keratometric, pachymetric, and elevation parameters of keratoconic corneas with Pentacam. Cornea 2009;28(9):976-980.

13. Quisling S, Sjoberg S, Zimmerman B, Goins K, Sutphin J. Comparison of Pentacam and Orbscan IIz on posterior curvature topography measurements in keratoconus eyes. Ophthalmology 2006;113(9):1629-1632.

14. Emre S, Doganay S, Yologlu S. Evaluation of anterior segment parameters in keratoconic eyes measured with the Pentacam system. J Cataract Refract Surg 2007;33(10):1708-1712.
15. Piñero DP, Alió JL, Alesón A, Vergara ME, Miranda M. Corneal volume, pachymetry and correlation of anterior and posterior corneal shape in subclinical and different stages of clinical keratoconus. J Cataract Refract Surg 2010;36(5):814-825.

16. Barr JT, Wilson BS, Gordon MO, Rah MJ, Riley C, Kollbaum $\mathrm{PS}$, Zadnik K. Estimation of the incidence and factors pre-dictive of corneal scarring in the Collaborative Longitudinal Evaluation of Keratoconus (CLEK) Study. Cornea 2006;25(1):16-25.

17. McMahon TT, Edrington TB, Szczotka-Flynn L, Olafsson HE, Davis LJ, Schechtman KB. Longitudinal changes in corneal curvature in keratoconus. Cornea 2006;25(3):296-305.

18. Ishii R, Kamiya K, Igarashi A, Shimizu K, Utsumi Y, Kumanomido T. Correlation of Corneal Elevation With Severity of Keratoconus by Means of Anterior and Posterior Topographic Analysis. Cornea 2012;31(3):253-258.

19. Altman DG, Bland JM. Diagnostic tests 3: receiver operating characteristic plots. BJM 1994;309(6948):188.

20. David Smadja, Santhiago MR, Mello GR, Krueger RR, MSE; Colin J, Touboul D. Influence of the reference surface shape for Discriminating between Normal Corneas, Subdimical Keratoconus and keratoconus. J Refract Surg 2013;29(4):274-281.

21. Ucakhan OO, Cetinkor V, Ozkan M, Kanpolat A. Evaluation of Scheimpflug imaging parameters in subclinical keratoconus, keratoconus, and normal eyes. Journal of cataract and refractive surgery 2011;37(6):1116-1124.

22. Henriquez MA, Izquierdo L Jr, Mannis MJ. Intereye asymmetry detected by Scheimpflug imaging in subjects with normal corneas and keratoconus. Cornea 2013 Jun;32(6):779-782. 